\title{
Antiplatelet effects of micronized fenofibrate in subjects with dyslipidemia
}

\author{
Anetta Undas ${ }^{1,2}$, Magdalena Celińska-Löwenhoff' \\ 1 II Department of Internal Medicine, Jagiellonian University School of Medicine, Kraków, Poland \\ ${ }^{2}$ Institute of Cardiology, Jagiellonian University School of Medicine, Kraków, Poland
}

\begin{abstract}
Introduction. Fibrates produce additional actions such as reduction of inflammatory state, insulin resistance and activation of blood coagulation, along with stimulation of fibrinolysis. It is not known whether fibrates can attenuate platelet activation. Objectives. Evaluation of antiplatelet effects of fenofibrate in dyslipidemic subjects. Patients and methods. In 20 patients ( 15 males, 5 females) aged 40 to 70 years who had plasma triglycerides $>1.7 \mathrm{mmol} / \mathrm{l}$ and low-density lipoprotein (LDL) cholesterol $>3.4 \mathrm{mmol} / \mathrm{l}$ without diabetes, we determined plasma levels of platelet markers, soluble CD40 ligand (sCD40L) and P-selectin, both in peripheral blood and samples collected every 1 minute from sites of microvascular injury prior to and following a onemonth administration of micronized fibrate $(160 \mathrm{mg} / \mathrm{d})$. Results. Neither of platelet activation markers was altered following fenofibrate. We identified 7 subjects who had a significant decrease $(14-21 \%)$ in velocity of the SCD4OL and $\mathrm{P}$-selectin release after fenofibrate $(\mathrm{p}<0.05)$. This subgroup was characterized by increased body mass, and posttreatment greater reduction in triglycerides and increase in high-density lipoprotein (HDL) cholesterol $(p<0.05)$. A decrease in the release of platelet markers was associated with a greater posttreatment reduction in plasma 8 -isoprostane levels $(p=0.006)$. Conclusions. In $1 / 3$ of dyslipidemic subjects without diabetes, there is a decrease in platelet activation at the site of microvascular injury following a one-month administration of micronized fenofibrate. This effect can be found in individuals in whom the fibrate induced the greatest reduction in triglycerides and increase in HDL cholesterol. Moreover, antiplatelet effect of fenofibrate was associated with reduced oxidative stress.
\end{abstract}

Key words: dyslipidemia, fibrates, isoprostanes, platelets

\section{INTRODUCTION}

Fibrates represent a class of drugs being synthetic ligands for the peroxisome proliferator-activated receptor- $\alpha$ (PPAR- $\alpha$ ), which can be abundantly found, e.g. in muscles, adipose tissue, liver and vascular wall, and their activation modifies transcription of numerous genes participating in processes such as lipid metabolism and inflammation [1]. By increasing lipoprotein lipase activity, apolipoprotein A1 production and reduction of apolipoprotein CIII secretion, fibrates stimulate the catabolism of triglyceride-rich lipoproteins. Therefore, the basic effect of fibrates consists in the reduction of plasma concentration of triglycerides by $30-50 \%$ and the increase in concentration of high-density lipoprotein cholesterol (HDL) by $2-20 \%$, whereas the effect of those drugs on concentration

Correspondence to:

dr hab. med. Anetta Undas, Instytut Kardiologii, Collegium Medicum Uniwersytetu Jagiellońskiego, ul. Prądnicka 80, 31-202 Kraków, Poland, phone: +48-614-30-04, fax: +48-423-39-00, e-mail: mmundas@cyf-kr.edu.pl

Received: July 31, 2007. Accepted in final form: August 3, 2007.

Conflict of interest: none declared.

Pol Arch Med Wewn. 2007; 117 (5-6): 235-240

Copyright by Medycyna Praktyczna, Kraków 2007 of low-density lipoprotein cholesterol (LDL) is variable - from neutral to slight reduction, usually by up to $10 \%$ [1-3]. Growing evidence indicates that fibrates have potentially beneficial additional effects, being able to reduce development of atherosclerosis, such as reduction of inflammatory state, insulin resistance and activation of coagulation, angiogenesis modulation and increased fibrinolytic activity [2-5]. Since 1974 there have also been reports suggesting that by lipid profile improvement, PPAR- $\alpha$ antagonists may attenuate platelet activation, resulting in impairment of platelet aggregation [6, 7 ], however, other investigators failed to demonstrate such an effect in humans; they even claimed that fibrates, like gemfibrozil and ciprofibrate, increase platelet activity [8, 9]. It is also unclear which factors can determine diverse effects of fibrates on platelet activation in dyslipidemic patients.

The objective of the study was to assess the effect of micronized fenofibrate on the release of platelet markers at the site of haemostatic plug formation after vascular injury in high cardiovascular risk non-diabetic patients, who do not have high hypertriglyceridemia. It was also tested whether fenofibrate administration in those patients may have any impact on oxidative stress, usually associated with dyslipidemia. 


\section{PATIENTS AND METHODS}

The prospective study involved 20 patients aged 40 to 70 years. Inclusion criteria were LDL cholesterol levels $>3.4$ $\mathrm{mmol} / \mathrm{l}$, triglyceride concentration $>1.7 \mathrm{mmol} / \mathrm{l}$ and being a patient from a high risk group according to the SCORE system. The criteria excluding from the study were diabetes, defined as taking hypoglycemic drugs, insulin therapy or fasting glycemia $>6 \mathrm{mmol} / \mathrm{l}$; hypolipemic treatment within 2 months prior to the study; uncontrolled hypertension; renal insufficiency (creatinine concentration $>177 \mathrm{mmol} / \mathrm{l}$ ); liver damage (alanine aminotransferase level $>1.5$ times higher than the upper limit of the reference range); malignancy or other serious disease; a history of recent infection or acute coronary syndrome at least 6 months earlier. We assessed platelet activation prior to administration of micronized fenofibrate (Lipanthyl Supra, Fournier) and after the 4-week administration of this drug in a daily dose of $160 \mathrm{mg}$ [10]. All patients involved in the study were taking acetylsalicylic acid in a daily dose of $75 \mathrm{mg}$. Other agents, like beta-blockers, angiotensin-converting enzyme inhibitors and diuretics, were used during the study in unchanged doses.

The study was approved by the Bioethical Committee of the Jagiellonian University. The patients gave their written informed consent.

The tests were carried out directly prior to administration of the first dose and after a month's treatment. Lipid profile, platelet count, creatinine, alanine aminotransferase and glucose levels were determined by routine tests in the hospital laboratory. Concentrations of fibrinogen and C-reactive protein (CRP) were determined by means of nephelometry (Dade Behring). In order to evaluate the intensity of oxidative stress, 8-iso-prostaglandin $\mathrm{F}_{2 \alpha}$ (8-iso-PGF $\left.{ }_{2 \alpha}\right)$, a product of non-enzymatic peroxidation of arachidonic acid, was also measured (Cayman Chemicals). Plasma concentrations of thromboxane $\mathrm{B}_{2}$ was determined twice (Cayman Chemicals) to prove the patients had taken acetylsalicylic acid. Prior to and after fibrate treatment, this concentration in all patients was typical of ones treated with acetylsalicylic acid and did not exceed $1 \mathrm{ng} / \mathrm{ml}$ (on average 0.3).

Platelet activation was assessed by determination of soluble CD40 ligand (sCD40L) and P-selectin levels in peripheral blood plasma taken at minimum venous stasis and in supernatants obtained from blood collected from standard forearm skin incisions made using a disposable Simplate II bleeding time device (Organon Teknika). The model of microvascular injury was discribed in detail previously [11-13]. Changes in concentrations of markers measured in subsequent blood samples were described by calculation of the following parameters: maximum velocity of concentration increase and maximum concentration in a one-minute sample, considering the amount of an analyte in the sample. The analysis was limited to the first 5 minutes of bleeding. Both markers were determined by means of commercially available immunoenzymatic tests according to instructions of the manufacturer, diluting the su- pernatant 10 to 100 times from the second minute. Variability of the parameters studied was about $7 \%$.

\section{Statistical analysis}

The data were presented as the mean and the standard deviation or the median (interquartile range). The Kolmogorov-Smirnov test was used to test whether the variables were normally distributed. The pre-treatment and post-treatment results were analyzed by means of the Wilcoxon test. Comparisons within the group were carried out by means of the MannWhitney U test. The Spearman's Rank Correlation Coefficient was calculated to assess relations between the variables, which were not normally distributed. A p-value of $<0.05$ was considered statistically significant.

\section{RESULTS}

As presented in the table, the study involved 20 subjects, all of whom completed the study. No significant adverse effects were observed. The patients were overweight, had slight or moderate hypercholesterolemia and slight hypertriglyceridemia, as well as low-grade inflammatory reaction with the CRP level exceeding $1 \mathrm{mg} / \mathrm{l}$ in all of them. As expected, after a month-long administration of micronized fenofibrate, a significant reduction of triglyceride concentration and an increase in the HDL cholesterol level were found (see table). No changes in total and LDL cholesterol, and fibrinogen were observed. The plasma CRP level was significantly lower. The concentration of sCD40L in venous blood decreased significantly, whereas peripheral blood concentration of P-selectin was not changed by administration of micronized fenofibrate. Bleeding time after a month's administration was significantly prolonged, on average, by $45 \mathrm{~s}$ (see table).

A profile of the release of the analyte amount, considering the sample volume as a function of bleeding time, was similar for sCD40L and P-selectin both before and after a month's treatment with micronized fenofibrate, demonstrating a phase of a rapid increase and plateau (fig.). Levels of both platelet markers measured in 1-minute intervals were strongly correlated prior to the commencement of treatment with micronized fenofibrate $(r=0.72 ; p<0.001)$ and after a month $(r=0.77$; $\mathrm{p}<0.001)$. No correlation between the maximum velocity of marker release or their maximum levels and the lipid profile, age, the CRP level or other assessed parameters was found. The analysis of the whole group $(n=20)$ demonstrated that in the microvascular injury model a month-long use of micronized fenofibrate was related to the reduction in neither maximum velocity of $\mathrm{sCD} 40 \mathrm{~L}$ or $\mathrm{P}$-selectin release, nor maximum concentration of both markers (see table and fig. A-B).

A tercile distribution of a variable, a degree of change of maximum velocity of platelet marker release, was applied to identify patients, in whom fenofibrate could reduce platelet activation expressed in a quantity of proteins released from plate- 


\begin{tabular}{|c|c|c|c|}
\hline \multicolumn{4}{|c|}{ Table. The study group characteristics before and after a month's treatment with micronized fenofibrate in $160 \mathrm{mg} / \mathrm{d}$ dose $(\mathrm{n}=20$} \\
\hline BMI $\left(\mathrm{kg} / \mathrm{m}^{2}\right)$ & $28.2(25.4-30.8)$ & - & - \\
\hline Smoking, $\mathrm{n}(\%)$ & $10(50)$ & - & - \\
\hline Hypertension, $n(\%)$ & $12(60)$ & - & - \\
\hline History of Ml, $\mathrm{n}(\%)$ & $10(50)$ & - & - \\
\hline $\mathrm{TC}(\mathrm{mmol} / \mathrm{l})$ & $6.48(5.57-7.12)$ & $6.22(5.46-6.72)$ & NS \\
\hline LDL cholesterol (mmol/l) & $3.87(3.52-4.19)$ & $3.78(3.43-3.96)$ & NS \\
\hline $\mathrm{HDL}$ cholesterol (mmol/l) & $1.68(1.44-1.79)$ & $1.93(1.54-2.19)$ & $<0.0001$ \\
\hline Triglycerides (mmol/l) & $2.29(1.92-2.84)$ & $1.59(1.26-2.01)$ & $<0.0001$ \\
\hline Glucose (mmol/l) & $5.1(4.4-5.8)$ & $5.2(4.3-5.9)$ & NS \\
\hline Fibrinogen $(g / l)$ & $3.03(2.43-3.61)$ & $2.92(2.37-3.57)$ & NS \\
\hline Platelet count, $\times 10^{3} / \mu \mathrm{l}$ & $219.3(181.9-254.7)$ & $231.4(180.4-269.2)$ & NS \\
\hline $\mathrm{CRP}(\mathrm{mg} / \mathrm{l})$ & $2.59(198-3.34)$ & $1.64(1.28-2.26)$ & $<0.0001$ \\
\hline sCD40L (ng/ml) & $1.52(1.18-1.82)$ & $0.63(0.47-0.85)$ & 0.004 \\
\hline P-selectin (ng/ml) & $105.4(78.5-129.6)$ & $113.2(88.8-127.5)$ & NS \\
\hline 8-iso-PGF ${ }_{2 \alpha}(\mathrm{pg} / \mathrm{ml})$ & $149.2(113.4-179.4)$ & $112.8(98.1-137.3)$ & $<0.0001$ \\
\hline $\mathrm{BT}(\mathrm{s})$ & 409 (381-449) & 454 (403-489) & 0.03 \\
\hline \multicolumn{4}{|c|}{ Maximum velocity of release of:* } \\
\hline $\mathrm{sCD} 40 \mathrm{~L}(\mathrm{pg} / \mathrm{s})$ & $1.31(1.06-1.67)$ & $1.24(1.01-1.45)$ & NS \\
\hline P-selectin (ng/s) & $0.21(0.16-0.27)$ & $0.20(0.14-0.25)$ & NS \\
\hline \multicolumn{4}{|c|}{ Maximum release in one minute ${ }^{*}$} \\
\hline sCD40L (pg) & $190.4(128.2-258.2)$ & $169.3(117.4-239.1)$ & NS \\
\hline P-selectin (ng) & $2.32(1.67-2.91)$ & $2.21(1.48-2.72)$ & NS \\
\hline \multicolumn{4}{|c|}{ * at the site of microvascular injury } \\
\hline \multicolumn{4}{|c|}{ 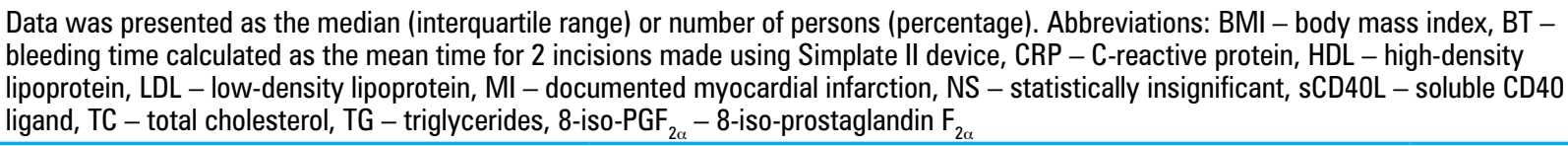 } \\
\hline
\end{tabular}

lets. A group of 7 patients, who demonstrated post-treatment reduction in the velocity of $\mathrm{sCD} 40 \mathrm{~L}$ and $\mathrm{P}$-selectin release at the site of vascular injury by $14-21 \%$, median $17 \%$ ( $\mathrm{p}<0.05)$, was found. It was presented in fig. C-D, and the aforementioned change varied significantly from a corresponding value calculated for the remaining patients $(p<0.001)$. Thirteen patients of the remaining 2 terciles demonstrated similar velocity of platelet marker release before and after treatment (median velocity change after treatment for the lower tercile $+3 \%$, and for the middle one $-5 \%$ ). In comparison with the patients belonging to the middle and lower terciles of reduction degree, 7 persons from the highest tercile were characterized by higher BMI (29.3 [27.4-31.8] vs. 26.9 [24.4-28.3] kg/ $\left.\mathrm{m}^{2}, \mathrm{p}=0.03\right)$, higher pre-treatment triglyceride levels $(2.69$ [2.22-2.93] vs. $2.01[1.58-2.24] \mathrm{mmol} / \mathrm{l}, \mathrm{p}=0.01)$, and after treatment, by the highest decrease in triglyceride levels $(0.82$ $[0.65-1.08]$ vs. $0.67[0.54-0.81] \mathrm{mmol} / \mathrm{l}, \mathrm{p}=0.03)$ and the highest increase in HDL cholesterol levels (0.36 [0.24-0.44] vs. $0.31[0.22-0.42] \mathrm{mmol} / \mathrm{l}, \mathrm{p}=0.03)$. Significant reduction in the release rate of both platelet markers at the site of microvascular injury for the patients of the higher tercile was also associated with reduction in the intensity of oxidative stress expressed by plasma 8-isoprostane levels (reduced by 55.1 [61.1-44.7) vs. $34.2(29.3-47.9) \mathrm{pg} / \mathrm{ml}, \mathrm{p}=0.006)$. Patients from the higher tercile did not differ from the remaining ones in respect of other analyzed parameters, both clinical, including risk factors and administered drugs, and laboratory, including plasma sCD40L and P-selectin concentrations (data not shown). A tercile analysis for the other variable describing the curve, i.e. the maximum amount of studied protein in a minute interval, showed that 6 of 7 patients from the highest tercile for release velocity were also found in the higher tercile for current division. 


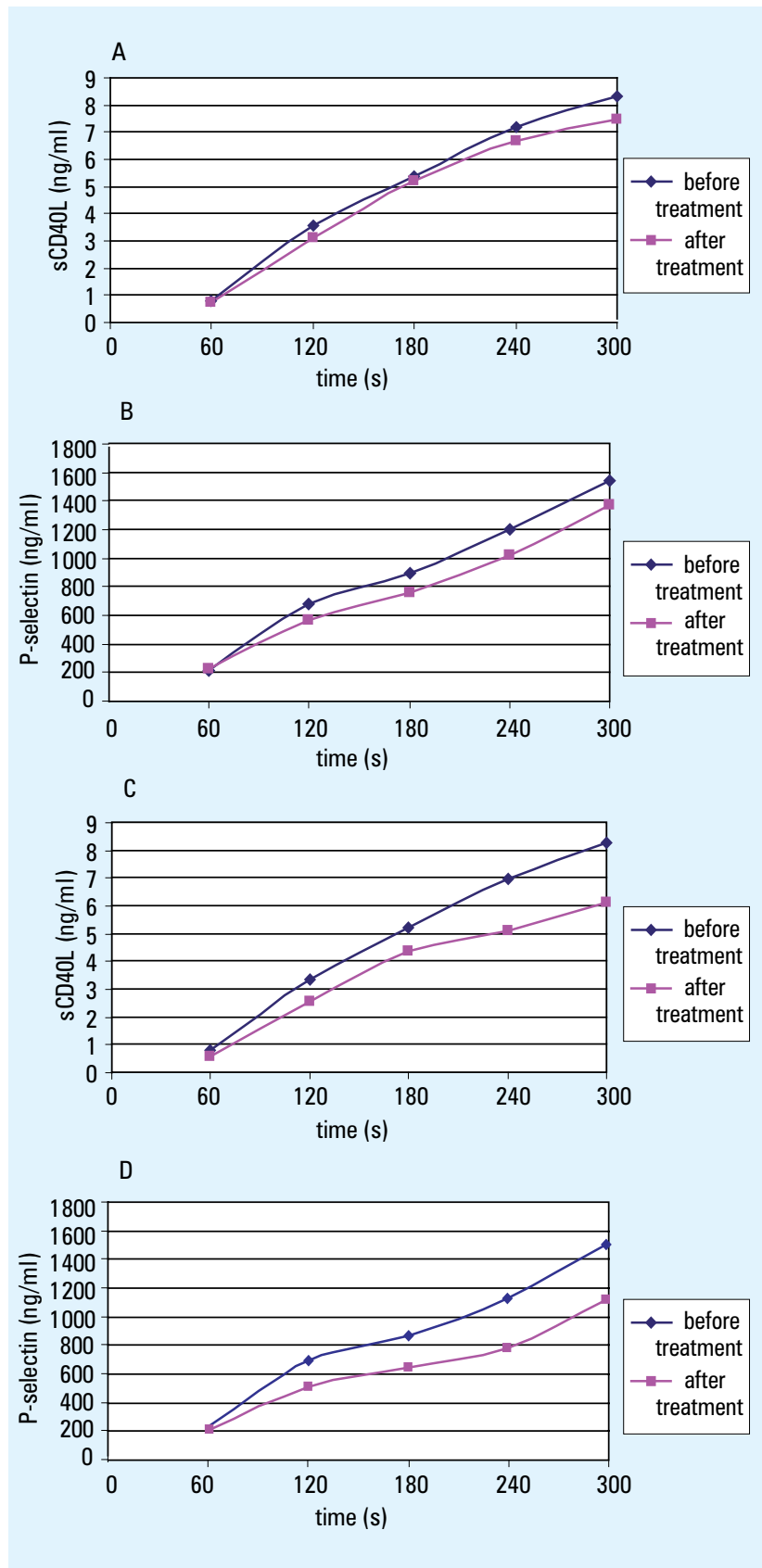

Fig. Course of release of soluble ligand CD40 (sCD40L) and P-selectin at the site of vascular injury prior to fenofibrate administration $(160 \mathrm{mg} / \mathrm{d})$ and after the 28-day administration. A-B. The whole group of 20 patients. C-D. Data for 7 patients of the higher tercile of persons, who demonstrated the highest reduction in velocity of platelet marker release. Data were presented as the medians

\section{DISCUSSION}

In previous publications we demonstrated that, as opposed to statins, fenofibrate did not reduce platelet activation assessed based on platelet granule protein, like $\beta$-thromboglobulin or sCD 40L, at the site of vascular injury, although there were ob- served subjects who demonstrated such a reduction, indicating that a possible antiplatelet effect of fenofibrate is characteristic only of some patients $[11,14]$. The current results suggest that in every third patient with slight hypercholesterolemia and hypertriglyceridemia there is a substantial impairment of platelet activation, expressed in the reduction in platelet granule protein release only after a month's treatment with micronized fenofibrate. Such a beneficial effect of the drug has been found in patients with the greatest changes in triglycerides and HDL cholesterol levels. Despite normal glycemia, the sujects in whom impairment of platelet activity was found after fenofibrate treatment, fulfilled the criteria for metabolic syndrome. This observation shows that patients with hyperglycemia could similarly respond to fibrate treatment. Contrary to the observations of Ferroni et al. [15], the antiplatelet effect of fenofibrate had no relation to reduction in the LDL cholesterol level. The antiplatelet effect was not also associated with CRP levels in the studied group. It is worth emphasizing that antiplatelet effect of micronized fenofibrate occurred in persons subject to chronic administration of acetylsalicylic acid having a well-known effect of platelet reactivity.

Impact of micronized fenofibrate on the release of platelet markers is probably variable, as suggested by our observations, according to which the sCD40L level in peripheral blood is subject to reduction during treatment, while the concentration of soluble P-selectin remains unchanged, despite the fact that in the model of vascular injury both markers show a strong relation and their levels are reduced not in all patients. Wang et al. [16] showed a significant reduction in sCD40L levels after 8 weeks of fenofibrate treatment. In available literature, there is no data concerning possible impact of the drug on blood P-selectin levels. Reasons for the differences in changes of sCD40L and P-selectin levels after the use of micronized fenofibrate are unclear. Soluble CD $40 \mathrm{~L}$ is released not only from platelets, but also from endothelial cells, monocytes and activated T cells, however, 95\% of its circulating pool is of platelet origin, which distinguishes $\mathrm{sCD} 40 \mathrm{~L}$ from P-selectin, being of endothelial origin to a greater extent [17]. In the microvascular injury model, proteins released from platelets are undoubtedly predominant. It therefore seems that some post-drug effects may be revealed only at the site of platelet activation; they may vanish, if the analysis consists only in assessment of venous blood sample taken from a peripheral vessel.

The observed changes of other parameters assessed in our study require a commentary. After a month-long administration of micronized fenofibrate we observed a significant reduction in the CRP level, considered as a major marker of inflammatory state. Wang et al. [16] found a substantial reduction in the CRP level, determined using a high-sensitivity method, after an 8-week treatment in a group with daily administration of $200 \mathrm{mg}$ of micronized fenofibrate. Similar observations were made by other researchers [18, 19]. Delerive et al. [20] also proved reduction in interleukin 6 levels in serum of patients undergoing a 4 -week fenofibrate treatment. Post-treatment reduction in the CRP level is then most likely the result 
of a similar change in production of cytokine controlling its synthesis, as indicated by the results of our study on micronized fenofibrate [11]. Moreover, in our study fenofibrate did not reduce fibrinogen concentrations. Available data on this issue are inconsistent. Previously, it was found that the agent has no impact on plasma fibrinogen concentration or induces usually its slight reduction $[2,19,21,22]$. Due to the fact that interleukin 6 has a strong impact on fibrinogen concentration, it seems that other modifying factors may be responsible for lack of reduction in its concentration observed by us [11].

What is interesting is our new observation, showing that micronized fenofibrate may substantially reduce oxidative stress, as showed by the reduction in plasma 8-iso-PGF ${ }_{2 \alpha}$ levels by over $20 \%$ after a month's administration of the agent. Recently Arca et al. [23] demonstrated that another oxidative stress marker, i.e. oxysterol, which occurs in the increased concentration in patients with familiar hyperlipidemia, is considerably reduced, when fenofibrate or atorvastatin is administered. Fenofibrate may also show properties such as increased antioxidative activity of glutathione peroxidase [24]. In our study isoprostanes were selected as an indicator of oxidative stress, successfully determined in plasma or urine by means of liquid chromatography or one of several immunoenzymatic methods [25]. Plasma concentrations measured in the study were slightly lower than the ones found by means of the same method in coronary patients [26]. Since oxidative stress undoubtedly contributes to the development of atherosclerosis [25], reduction in this process caused by fenofibrate may, irrespective of its effect on platelets, constitute a significant mechanism of beneficial effect of this drug.However, such an effect in diabetic patients has not been proved yet.

A small number of the analyzed group is the most significant limitation of the current study. Moreover, it is not clear whether the same effects could be seen in diabetics or patients with high triglyceride levels or low HDL cholesterol levels, because such patients were not included in the current study. Our observations, however, concern a larger group of people at high cardiovascular risk, with moderately increased cholesterol levels, slight triglyceridemia and mild to moderate arterial hypertension, and overweight, smoking patients, who might benefit from fenofibrate therapy. Such a hypothesis requires, however, a study with a long-term follow-up and assessment of clinically significant outcomes.

In conclusion, the study showed that micronized fenofibrate is able to reduce platelet activation in high risk, nondiabetic patients, who are overweight and who demonstrate a significant reduction in triglyceride levels and an increase in HDL cholesterol levels. Antiplatelet effects of this drug are associated with attenuation of oxidative stress. This new, so-called pleiotropic, effect of fenofibrate indicates that some therapeutic benefits of fenofibrate administration may be related to impairment of systemic peroxidation during the therapy. A fact that impairment of platelet activation observed during fenofibrate treatment concerns only some patients, may also partly explain inconsistent results of fibrate clinical studies and indicate the need for individualization of treatment with this class of drugs [27]. It is essential to perform further large studies, involving also subjects diagnosed with diabetes - a large subgroup of patients contemporarily treated with fibrates, in order to validate our observations and their potential clinical implications.

\section{ACKNOWLEDGMENTS}

The study was supported by the Ministry of Science - project No. 3PO5B 16322. The authors wish to thank Fournier Laboratoires for providing the drug for the study.

\section{REFERENCES}

1. Forman BM, Chen J, Evans RM. Hypolipidemic drugs, polyunsaturated fatty acids, and eicosanoids are ligands for peroxisome proliferator-activated receptors alpha and delta. Proc Natl Acad Sci USA. 1997; 94: 4312-4317.

2. Han SH, Quon MJ, Koh KKZ. Beneficial vascular and metabolic effects of peroxisome proliferator-activated receptor-alfa activators. Hypertension 2005; 46: 1086 1092.

3. Israelin-Konaraki Z, Reaven PD. Peroxisome proliferator-activated receptor-alfa and atherosclerosis: from basic mechanisms to clinical implications. Cardiol Rev. 2005; 13: $240-246$.

4. Chinetti-Gbaguidi G, Furchart JC, Steals B. Pleiotropic effects of fibrates. Curr Atheroscler Rep. 2005; 7: 396-401.

5. Okopień B, Łebek M, Herman ZS. Plejotropowe działania fibratów. Pol Arch Med Wewn. 2001; CV1: 1187-1191.

6. Carvalho ACA, Colman RW, Lees RC. Clofibrate reversal of platelet hypersensitivity in hyperbetalipoproteinemia. Circulation 1974; 50: 570-574.

7. Pazzucconi $F$, Mannucci $L$, Mussoni $L$, et al. Bezafibrate lowers plasma lipids, fibrinogen, and platelet aggregability in hypertriglyceridemia. Eur $\mathrm{J}$ Clin Pharmacol. 1992; 43: 219-223.

8. Broijerson A, Eriksson M, Angeli B, et al. Gemfibrozil enhances platelet activation in patients with combined hyperlipoproteinemia. Arterioscler Thromb Vasc Biol. 1995; 15: 121-127.

9. Gajdos M, Mongiellova V, Huttova D, et al. Ciprofibrate increases plasma concentration of platelet-derived growth factor $A B$ in patients with advanced atherosclerosis and hyperlipidemia independently of its hypolipidemic effects. J Cardiovasc Pharmacol. 2001; 38: 651-656.

10. Keating GM, Ormrod D. Micronised fenofibrate: an updated review of its clinical efficacy in the management of dyslipidaemia. Drugs 2002; 62: 1909-1944.

11. Undas A, Celinska-Lowenhoff $M$, Domagała TB, et al. Early antithrombotic and antiinflammatory effects of simvastatin versus fenofibrate in patients with hypercholesterolemia. Thromb Haemost. 2005; 94: 193-199.

12. Undas A, Brummel KE, Musial J, et al. Simvastatin depresses blood clotting by inhibiting activation of prothrombin, factor $\mathrm{V}$, and factor XIII and by enhancing factor Va inactivation. Circulation 2001; 103: 2248-2253.

13. Undas A, Brummel K, Musial J, et al. Blood coagulation at the site of microvascular injury: effects of low-dose aspirin. Blood 2001; 98: 2423-2431.

14. Undas A, Stępień E, Nizankowski $R$, et al. Effects of simvastatin on angiogenic growth factors released at the site of microvascular injury. Thromb Haemost. 2006 96: 342-347.

15. Ferroni P, Basili S, Santilli F, et al. Low-density lipoprotein-lowering medication and platelet function. Pathophysiol Haemost Thromb. 2006; 35: 346-354.

16. Wang TD, Chen WJ, Lin JW, et al. Efficacy of fenofibrate and simvastatin on endothelial function and inflammatory markers in patients with combined hyperlipidemia: relations with baseline lipid profiles. Atherosclerosis 2003; 170: 315-323.

17. Anand SX, Viles-Gonzalez JF, Badimon JJ, et al. Membrane-associated CD40L and sCD40L in atherothrombotic disease. Thromb Haemost 2003; 90: 377-384.

18. Koh KK, Han SH, Quon MJ, et al. Beneficial effects of fibrate to improve endothelia dysfunction and raise adiponectin levels in patients with primary hypertriglyceridemia. Diabetes Care 2005; 28: 1419-1424.

19. Okopień B, Krysiak R, Herman ZS. Effects of short-term fenofibrate treatment on circulating markers of inflammation and hemostasis in patients with impaired glucose tolerance. J Clin Endocrinol Metab. 2006; 91: 1770-1778. 


\section{ORIGINAL ARTICLES}

20. Delerive P, De Bosscher K, Besnard S, et al. Peroxisome proliferator-activated receptor alpha negatively regulates the vascular inflammatory gene response by negative cross-talk with transcription factors NF-kappaB and AP-1. J Biol Chem 1999; 27: 32048-32054.

21. Okopień $B$, Cwalina $L$, Lebek $M$, et al. Effects of fibrates on plasma prothrombotic activity in patients with type Ilb dyslipidemia. Int. J Clin Pharmacol Ther. 2001; 39: 551-557.

22. Maison $P$, Mennen $L$, Sapinho D, et al. A pharmacoepidemiological assessment of the effect of statins and fibrates on fibrinogen concentration. Atherosclerosis. 2002 160: $155-160$.

23. Arca M, Natoli S, Micheletta F, et al. Increased plasma levels of oxysterols, in vivo markers of oxidative stress, in patients with familial combined hyperlipidemia: reduction during atorvastatin and fenofibrate. Free Radic Biol Med. 2007; 42: 698705.

24. Tkac I, Molcaniova A, Javorsky $M$, et al. Fenofibrate treatment reduces circulating conjungated diene level and increases glutathione peroxidase activity. Pharmaco Res. 2006; 53: 261-264.

25. Patrono C, Falco A, Davi G. Isoprostane formation and inhibition in atherothrombosis. Curr Opin Pharmacol. 2005; 5: 198-203.

26. Vassalle C, Botto N, Andreassi MG, et al. Evidence of enhanced 8-isoprostane plasma levels, as index of oxidative stress in vivo, in patients with coronary artery disease. Coron Artery Dis. 2003; 14: 213-218.

27. Wierzbicki AS. Fibrates after the FIELD study: some answers, more questions. Diabetes Vasc Dis Res. 2006; 3: 166-171. 